\title{
EFECTOS (POS-)TEORÍA Y REFLEXIVIDAD EPISTEMOLÓGICA
}

\author{
Arturo CASAS \\ Universidade de Santiago de Compostela \\ arturo.casas@usc.es
}

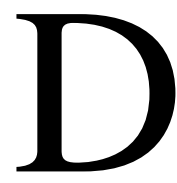
esde el reconocimiento intelectual y un profundo afecto, me gustaría compartir en estas páginas algunas consideraciones sobre un asunto por el que Enric Sullà ha mostrado permanente interés como investigador, analista y docente universitario, según deja ver muy a las claras, por ejemplo, la reciente publicación en Els Marges de un artículo de sugerente e intrigante título, «I ara, què fem? Breu historia de la Teoria (de la Literatura)» (Sullà, 2013). Se trata de cuestiones que me planteo también desde hace tiempo, supongo que como cualquier colega preocupado, en el sentido más honorable de la expresión, por lo nuestro. Debido a ello enlazaré en lo que sigue reflexiones que me han acompañado durante años de actividad académica con otras más recientes, varias de ellas promovidas por la lectura de los trabajos del compañero que ahora se jubila.

\section{De un ocaso a otro}

Visto en perspectiva, el camino recorrido por la teoría literaria y cultural desde el ocaso del estructuralismo constituye una sucesión no lineal de encrucijadas que consiguió abrir de forma apreciable alternativas epistemológicas y propició además sucesivos vínculos y diálogos con diferentes ramas del saber, de modo que para bien o para mal la lingüística estructuralista de fundamentación no pragmática dejó de ser la ciencia privilegiada - casi única en algún momento- de interlocución interdisciplinar e incluso, por esa misma pulsión, de afianzamiento y legitimación de una cientificidad tan largamente autoexigida como mal gestionada. Siendo esto así, parece certera, simultáneamente, la percepción de Terry Eagleton (2004: 13) cuando indica que en el desarrollo de ese proceso de poco más de medio siglo han terminado por volatilizarse la ambición y la originalidad de los promotores iniciales del pensamiento teórico moderno sobre la literatura y la cultura. Vale la pena recuperar la forma exacta en la que Eagleton inicia After Theory. Cito por la traducción castellana y llamo la atención sobre la nómina de teóricos habilitada:

La edad de oro de la teoría cultural se terminó hace mucho tiempo. Los trabajos pioneros de Jacques Lacan, Claude Lévi-Strauss, Louis Althusser, Roland Barthes y Michel Foucault han quedado a varias 


\section{Arturo Casas}

décadas de nosotros. Así también le ha sucedido a los primeros innovadores escritos de Raymond Williams, Luce Irigaray, Pierre Bourdieu, Julia Kristeva, Jacques Derrida, Hélène Cixous, Jürgen Habermas, Fredric Jameson y Edward Said. Desde entonces no se ha escrito demasiado que haya igualado la ambición y originalidad de estos padres y madres fundadores (Eagleton, 2004: 13).

La senda antes aludida situó en primer plano, por otra parte, el carácter inestable de los objetivos de conceptualización y la propia delimitación de la teoría en cuanto teoría-de-algo no plenamente discernido. Un algo que dejó en suspenso la tradicional prefiguración ontológica y/o axiológica del objeto teórico y fomentó una negociación aparentemente indomable de heurísticas, dando entrada así a imprescindibles llamadas de atención desde el análisis del discurso, la culturología, la sociología, la ciencia política y las distintas formulaciones contemporáneas de la teoría crítica, sin por ello desentenderse de manera obligada de la estética, por ejemplo. Se recordará a este respecto que ya la Escuela de Praga, a través de Mukařovský, Vodička y otros teóricos, propuso esa comprensión a propósito de la obra artística y de su constitución y recepción en el tiempo histórico.

La dialéctica entre constitución heurística y ansiedad metodológica -viciosa por desmedida e incluso caníbal en la percepción de algunos observadores prestigiosos- se nutrió de este modo con el potencial imparable de su propia inercia. A estas alturas del siglo XXI el vértigo perdura, si bien los ritmos parecen otros. Y a veces, sobre ellos, se impone una especie de fatiga, a mi modo de ver presente por caso en el Todorov de La Littérature en péril (2007), tanto por lo referido a la atribución funcional de la teoría y la crítica literarias como a la de su objeto (a uno de ellos, si se me permite la puntualización), la literatura.

La dialéctica de la que hablamos confluyó, como mínimo a partir de los años ochenta del siglo pasado, con la emergencia de un sujeto teórico otro, plural y contradictorio, irreductible a todo precedente a partir ya de la declaración de una diferencia cultural o de otro signo explícitamente expuesta y con frecuencia autoanalizada. El propio vínculo entre ese sujeto y su específica enunciación mudó, dejando afectada la forma del discurso teórico, la prefiguración de sus destinatarios primarios y secundarios, el lugar institucional de la enunciación teórica, su metadiscursividad y metalenguaje y, en fin, las reglas de su particular concurrencia en diversos campos sociales, incluidos, y no en último lugar, el campo de producción ideológica (Sapiro, 2007) y el campo político en sus dimensiones local y mundial (Butler, Guillory y Thomas, 2000; Bleiker, 2012). Además, y sin confusión posible con la ruta emprendida en la órbita anglosajona por los Cultural Studies, ni tampoco estrictamente con la herencia semiótico-cultural procedente de Tartu, se han ido haciendo notar cada vez más alto voces importantes que animan a entender que lo literario es en realidad entendible como subzona de la cultura (el prefijo es solo dimensional) y que por consiguiente alguna relación nueva habrá que explorar entre los estudios que tradicionalmente se han venido ocupando de lo literario y de lo cultural ${ }^{1}$ (Bal, 1999; Even-Zohar, 2010). En todo caso, y sin salirnos de las páginas iniciales del libro ya citado de Eagleton, autor con el que dialoga en profundidad Enric Sullà, se hace perceptible que la opinión del autor de

\footnotetext{
${ }^{1}$ El comparatismo, sobre todo el más reciente, ha asumido este reto. Compruébese por ejemplo en el volumen coordinado por Mercè Picornell y Margalida Pons Literatura i cultura: aproximacions comparatistes (2009), en el que por cierto Enric Sullà retoma su extensa y fértil línea de investigación sobre el canon con el capítulo «La institució literària i el cànon» (33$60)$.
} 
After Theory sobre todo lo que estamos esquematizando es bastante representativa del análisis dominante entre las generaciones más curtidas de teóricos y analistas de la cultura, con independencia de su filiación formal-estructuralista, semiótica, sociológica o marxista.

A mi modo de ver, lo anticiparé, resulta impropio por exageradamente caricaturesco afirmar, como hace el reconocido pensador inglés, que «en las orillas más inhóspitas de la academia, el interés por la filosofía francesa ha dejado paso a la fascinación por el beso francés», o que «hay un interés entusiasta por los cuerpos copulando, pero no por los cuerpos trabajando» (Eagleton, 2004: 14). Y no se piense, leyendo estas frases, que estamos apenas ante exabruptos o deslices momentáneos; aunque sería injusto obviar en este momento los matices introducidos por Eagleton a lo largo de ese volumen y quizás todavía más en The Event of Literature, aparecido en su versión original inglesa en 2012. En este último libro reclama de hecho puntos de encuentro entre la teoría literaria y la filosofía de la literatura, saber y discurso este último mucho más próximo a la forma de hacer y de argumentar anglosajona que a la propiamente continental. En el prólogo al nuevo libro leemos esto que sigue en su versión traducida:

El poscolonialismo, la multiculturalidad, la sexualidad y los estudios culturales no están, sin duda, vírgenes de teoría. Tampoco datan simplemente del declive de aquellos otros enfoques anteriores [semiótica, posestructuralismo, marxismo y psicoanálisis]. Sucede más bien que han aflorado en toda su plenitud al rebufo de la teoría «pura» o la «alta» teoría a las que, en su mayor parte, han dejado atrás. Y no solo las han dejado atrás, de hecho, sino que han contribuido a desplazarlas. En ciertos aspectos, se trata de una evolución que hay que acoger de buen grado (Eagleton, 2012: 11).

A partir de todo ello, hablar de normalidad o de crisis a propósito de la disciplina que reconocemos aún como teoría literaria - posteoría en algunos foros ${ }^{2}$ - sería tarea tan fútil como enmarañada. Lo indiscutible es que, además de alcanzar mayor presencia académica, la teoría ha sabido incrementar su capacidad de diálogo y entendimiento con otras disciplinas humanísticas, filológicas y semióticas, empezando por las también viejas retórica e historia, contribuyendo así a orientar el bloque de saberes conocido como ciencias humanas hacia el marco amplio de las ciencias sociales. Y ello a pesar de dos factores de peso que es oportuno ponderar.

El primero es el cuestionamiento de la figura cultural y política del teórico en tanto intelectual, contemplado a la luz de una triple discusión, la dada en simultaneidad sobre el par intervencióndistanciamiento, sobre las estrategias de planificación y de actuación en el espacio público y, en último pero no menos relevante lugar, sobre el poder. Digamos por tanto que hablamos de análisis y de posiciones como las que bien podrían representar Régis Debray (1979) y Richard Rorty (1998), por ejemplo; o también de las atribuibles, de nuevo por simplificar las cosas, a un Aijaz Ahmad (1992), una Judith Butler (1997), un Itamar Even-Zohar (2010) y un Pierre Bourdieu (2003).

El segundo factor de peso, sin duda interconectado con el anterior, es la admisión de una

\footnotetext{
${ }^{2}$ Entre las marcas de lo que Fernando de Toro (1999) o Ivan Callus y Stefan Herbrechter (2004) describen como condición posteórica destacaré cinco: la legitimidad y la productividad de hacer teoría situando frente a frente epistemologías en conflicto, la institucionalización y la internacionalización del pensamiento teórico, su proyección sobre las interdependencias culturapolítica, la ruptura de los límites interdisciplinares y la incorporación de la producción teórica proveniente de espacios geoculturales e identitarios periféricos.
} 
disposición enunciativa nueva en el discurso teórico. Se manifiesta esta en dos planos. Uno es la búsqueda de otro vínculo o pacto ${ }^{3}$ con los lectores y asimismo con los objetos de análisis, descrito ese pacto a veces como critical intimacy. El otro es una muy diferente comprensión, dúctil, no logocéntrica, del metalenguaje y del cuerpo teórico conceptual, en línea con lo expuesto por Deleuze y Guattari (1991) sobre la mutabilidad, la sintacticidad y la contingencia de los conceptos que utilizamos para entendernos y para tratar de clarificar problemas. Esto ha sido expresado de forma bastante gráfica por Mieke Bal en un libro no por casualidad titulado Travelling Concepts in the Humanities con la frase «concepts resonate rather than cohere»(Bal, 2002: 52).

En perspectiva histórica, el afianzamiento de una centralidad relativa del discurso teóricoliterario dista de ser un acontecimiento insólito. Supone de algún modo una recuperación del protagonismo de una disciplina recién mencionada, la retórica; en particular, como repertorio sistematizado de loci y como ordenación de los géneros del discurso, en un segmento cronológico vastísimo en sus dimensiones y ampliado aún en el siglo XVIII por la consolidación de la estética y la hermenéutica como preludio de la intervención crítica en la esfera pública y a la vez como antesala de la vorágine historicista. Con todo, la centralidad de la teoría (en otros términos, su institucionalización) representa algo más en nuestro tiempo.

Sin necesidad de reconducir la cuestión a la incidencia que en especial en el mundo anglosajón ha alcanzado el saber teórico en las esferas académica y editorial, regidas en buena medida, como tanto insistió David Lodge (1990), por leyes de mercado bastante explícitas, debe reconocerse con John Guillory (1993) la importancia de la substitución del predominio del capital cultural de la vieja burguesía, inscrito en lo fundamental como saber positivista-historicista, en favor de la emergencia de una nueva clase profesional universitaria que cobró presencia y poder asentada en un saber técnico y en estrategias que, de acuerdo con el propio Guillory, bien podrían describirse como ejecutivoburocráticas, administradas e instrumentalizadas estas incluso desde núcleos consolidados de contrapoder o desde contra-espacios públicos (Aguiar e Silva, 1998-1999: 90; Fraser, 1992; GonzálezMillán, 2000: 63-104). De ahí proceden algunas diatribas contra la teoría, que en la academia norteamericana y en otras partes confluyen a veces de modo paradójico con declaraciones de signo bien distinto, entre ellas las hechas para recuperar una actitud lectora y para rescatar un ejercicio crítico libre, independientes supuestamente de todo principio coercitivo en el análisis y la interpretación de los textos (Goulart, 2001).

Y no como coerción pero sí como renuncia a cumplir un programa social y político efectivo -desde Horkheimer, el propio de una teoría crítica o una filosofía social-, es recurrente asimismo la refutación del autotelismo abstracto de la teoría, de un intelectualismo ineficaz y neutro en términos

\footnotetext{
${ }^{3}$ Patrick Charaudeau (2009) discute las virtualidades de términos como los que se acaban de emplear, que él propone reemplazar por el de contrato de comunicación, resaltando además que este requiere una competencia psico-sociolingüística por parte de los sujetos implicados, a lo cual añade: «como esta competencia supone, ella misma, por parte de estos sujetos, un reconocimiento recíproco del saber que está en juego (el propósito), de la relación de poder que se instaura entre ellos (las identidades) y de los comportamientos posibles (las instrucciones de los roles discursivos), se puede decir que la noción de contrato es constitutiva de la comunicación lingüística». Más adelante retomaremos estas anotaciones.
} 
políticos y, en fin, de lo que Pierre Bourdieu denominaba errores epistemocéntricos, promotores de una lógica teorética, escolástica, que se desentiende de cualquier concreción analítica (Calhoun 1995: 132-161). El asunto es complejo, no puede negarse. Lo obvio, por concretar, es que el discurso teórico, la función teoría, emerge en nuestro tiempo entre el maremágnum global de signos contra otra clase bien distinta de discursos y disciplinas, distintos por ejemplo de aquellos con los que entró en competencia Tomachevski cuando en 1925 sacó a luz pública su Poética. Y fijémonos, pensando a la vez en el formalista ruso y en un teórico como Eagleton, que todo ello comparece además no solo en una dimensión global sino también bajo coordenadas locales. Esto es importante tenerlo en cuenta para entender de forma cabal diatribas como las que acabamos de constatar en el autor británico, probablemente innecesarias, por así decirlo, en un país y en una academia como los nuestros ${ }^{4}$.

Menos normal o esperada que la mencionada referencialidad de la teoría, y por tanto más susceptible de evaluarse en cuanto indicio a la luz de otros parámetros, es la reiterada interpelación mutua entre el saber teórico-literario y otras esferas de las ciencias sociales y humanas. Jonathan Culler (1997), al analizar la teoría como género, y esto ha llamado asimismo la atención de Enric Sullà (2013: 136), ha llegado a cifrar en la performatividad interdisciplinar la característica más significativa del discurso teórico. De modo que en su criterio la teoría es lo que produce efectos (también) fuera de su marco original de planteamiento. Recuérdese, por abundar en la misma línea, que Pierre Bourdieu (1994: 27) señalaba en Marx al pensador que con mayor intensidad ha ejercido hasta nuestro tiempo un effet de théorie, que en su perspectiva sería además «un effet proprement politique» por cuanto mostraría y constituiría realidades antes no reconocidas como tales en el mundo social ${ }^{5}$. De hecho, como ha visto François Provenzano (2010: § 44-46) siguiendo él mismo la metodología propia de la sociología de los campos sociales, la teoría promueve procesos de consagración que se concretan al menos en los siguientes efectos:

1) La consécration par la théorie s'inscrit dans un jeu d'opposition à d'autres formes de consécration (institutionnelle, critique/historiographique, ou également théorique).

2) L'opération de conceptualisation, qui est le propre de l'énonciation théorique, induit une orientation axiologique particulière, qui valorise directement ou indirectement telle ou telle unité traitée par la théorie (directement: par validation du modèle défendu; indirectement: par invalidation du modèle défendu). Cette valorisation s'accompagne d'un surinvestissement théorique et d'effets rhétoriques de naturalisation.

3) La position du théoricien lui-même, au sein de son champ de pratiques, est elle-même en jeu dans l'opération de consécration.

\section{Estructura y acontecimiento}

\footnotetext{
${ }^{4}$ Solo un ejemplo: véase en la reciente Escatología de la crítica de Aullón de Haro (2013) contra quién y qué se argumenta tanto en una escala global como en la propiamente local (nacional, si se prefiere), esta última de manera no tan explícita aunque en cualquier caso evidente. El libro de Aullón de Haro articula por otra parte una crítica cultural de nuestra era en la que son apreciables ciertos puntos de convergencia diagnóstica -no así restauradora-con lo expuesto por Richard Sennett en The Culture of the New Capitalism (2006), en particular por lo referido a la ligereza y superficialidad de discursos y prácticas en unas coordenadas trazadas por y para el consumo y el mercado, incluido por supuesto el académico. Cuando empleo la expresión «hablar contra alguien y/o contra algo» lo hago desde la asunción de lo expuesto por Bourdieu en el capítulo «Caso práctico: ¿Cómo leer a un autor?» de sus Meditaciones pascalianas (1997: 113-122).

${ }^{5}$ Son observaciones estas que en algún modo confluyen asimismo en el potente pensamiento estético-político de Jacques Rancière (2000).
} 
Regresemos ahora brevemente a una cuestión ya mencionada para introducir algún matiz a la luz de lo que acabamos de ver. A propósito del vértigo expansivo de la teoría hay un aspecto controvertido de la intervención teórica, y de sus específicos efectos, que Pozuelo Yvancos ha referido en distintas ocasiones como síndrome de autofagia. La expresión es apropiada para describir la consunción de la teoría literaria en los límites especulativos y metadiscursivos de ella misma, situación originada tanto por la ausencia de un consenso epistemológico mínimo como por la necesidad subsiguiente de autojustificación o delimitación teorética frente a las otras posiciones concurrentes.

Podría decirse de un modo más llano: probablemente, en nuestros días, la teoría se lee poco pese a cultivarse, prodigarse y discutirse mucho. Se lee poco, acaso, por lo apuntado hace un momento. Porque falta aquella gran teoría aglutinadora de otrora (Skinner, 1985), o porque falta interés por lo que con Rorty (1989), y posteriormente con Butler, Laclau y Žižek (2000), cabría interpretar como la contingencia teorizadora propia y de los demás, de los (muchos otros) que militan en otra epistemología, son condicionados por otros habitus e intervienen sobre otra heurística. O en fin porque faltan, expresado ahora en la terminología del sociólogo portugués Boaventura de Sou sa Santos (2002), condiciones de inteligibilidad y trabajo de traducción que hagan accesibles la lógica y el saber (no solo teóricos) del otro 6 .

Toda esta situación podría inducir en algunas sensibilidades teorizadoras una nostalgia por el orden (formal-estructuralista) perdido. No sé si algo de esto mismo comparecerá en Eagleton (2012) cuando, recuperando ideas de Kenneth Burke, Paul Ricoeur, Étienne Balibar, Pierre Macherey, Umberto Eco y en particular de Fredric Jameson, asevera que hay un punto de convergencia en la multiplicidad de las teorías literarias. Consistiría, ha señalado, en el hecho de asumir casi todas ellas sin excepción «que la obra literaria es una estrategia» (Eagleton, 2012: 217; cursivas en el original), una estrategia que además presupone siempre una suerte de reescritura simbólica de un contexto cultural necesariamente textualizado e inequívocamente dirigida a un receptor (individual, colectivo), razones estas por las que la obra es hecho y acto, o estructura y acontecimiento (Eagleton, 2012: 244).

Sin embargo, y contra cualquier forma de nostalgia, comparecen hoy por hoy claves alentadoras que estará bien resaltar. La primera es que la apertura epistemológica de la que estamos hablando ha favorecido la dinamización intelectual y el reemplazo de algunos esquemas agotados, con raíces bien en la modernidad bien en la posmodernidad. Ello es muy perceptible en la delimitación axiológica del objeto teorizado, sea este la literatura o cualquier otro producto/repertorio cultural. Es indudable que estamos bien pertrechados para entender -si queremos- qué es la canonicidad, qué es la distinción, qué es el gusto. Sullà (1997: 64-66; 2013) ha problematizado en un sentido próximo les fronteres de la literatura, que en la última de las dos publicaciones reseñadas se presentan desde una vertiente complementaria, por entero ineludible en su doble carácter cultural y político:

Representar el cànon literari com una llista tancada i inamovible d'autors i obres, com una Bíblia, i fer la universitat responsable de la seva administració, com una església, va servir per desencadenar les canon wars que van encendre els campus de les universitats nord-americanes entre els anys 80 i 90, probablement

\footnotetext{
${ }^{6}$ Me he extendido un poco más sobre estas cuestiones en otro lugar (Casas, 2013).
} 
como a resposta a les demandes d'una societat multiètnica més sensible a les reivindicacions del feminisme i de les minories. La polèmica va saltar als mitjans de comunicació per la indiscutible dimensió politicocultural.

La segunda podría ser, y así lo ha señalado Manuel Gusmão (2004: 636) actualizando una fórmula cara a Roland Barthes, la potenciación de la praxis teórica entendida como «uma das forças da literatura». Y ello se está logrando en simultaneidad frente a quienes buscan la esencialización de la literatura y frente a quienes, en sentido opuesto, proclaman su simple disolución en un espacio no acotado de textualidades múltiples.

\section{La literatura y lo literario ante el discurso teoría}

Lo paradójico del caso es, como ha visto con sagacidad Culler (en Butler, Guillory y Thomas, 2000: 273-292), que, mientras que la literatura puede haber perdido su centralidad como objeto específico de estudio, las ciencias sociales y humanas han comenzado a percibir buena parte de sus objetos de análisis como propiamente literarios, pues «in the humanities and the humanistic social sciences everything is literary» (289).

Las páginas que siguen procurarán desarrollar un esbozo de lo que podría llamarse discurso teoría. Como hemos visto, los métodos y propuestas de análisis del hecho y del acontecimiento literarios en la etapa posestructuralista han modificado la perspectiva de la generalidad de los estudiosos en cuanto a los objetos, los problemas, las metas y los procedimientos disciplinares. Del mismo modo, se ha visto afectada la asimilación de la convivencia con metodologías alternativas e incluso con otros marcos de conocimiento, desplegándose con ello el encendido debate sobre el pluralismo y el relativismo neopragmatista (Duarte, 1989: 127-143), que lejos de cesar se ha reavivado en el cambio de siglo con la revisión del pluralismo dicho convencional y la exploración de un nuevo pluralismo, representable por ejemplo en la figura de William Connolly (Campbell y Schoolman, 2008).

Lo cierto es que una parte de las tensiones entre programas o escuelas se ha desplazado a otros planos por la propia multiplicación de los objetos de atención, ampliada exponencialmente por la pujanza de los estudios culturales, otra interdependencia entre teoría e historia literaria y también de un nuevo paradigma comparatista que trata de rehacerse frente al conocido augurio de Gayatri Spivak (2003) sobre la desaparición de la disciplina. En este sentido se hace capital no sólo la cuestión del sujeto teórico sino también, con ella, la del lugar de la enunciación teórica -lugar académico, lugar público, lugar institucional...- y su compleja dialéctica con los nuevos objetos de análisis visibilizados y con sus narrativas específicas (García Canclini, 2006: 147-166).

Al respecto, Siegfried J. Schmidt en «A Systems-Oriented Approach to Literary Studies» (1997) asociaba tres planos: 1) el de la relativización del objeto de estudio de la disciplina, 2) el cuestionamiento epistemológico de qué sea en realidad una teoría y 3) la improbabilidad de un ejercicio aséptico de la intervención teórica. El primero y el tercero refuerzan su convergencia desde el momento en que queda planteada la duda sobre la posibilidad de separar de forma nítida y completa 
sujeto y objeto de estudio, viejo debate del que ya se ocupaba Norbert Elias en 1956 en su célebre «Problems of Involvement and Detachment». Pero la cuestión no queda ahí, porque, como ha reconocido Pozuelo Yvancos (1999: 95), elegir una teoría en nuestro tiempo es mucho más que elegir un instrumental de análisis o un método: es fundamentalmente elegir el lugar desde el que definir un objeto.

¿Qué interés tiene lo anterior para nuestra indagación? Hace ya bastantes años Douwe Fokkema (en Angenot et al., 1989) esbozó una presentación crítica de la (in)disociabilidad de sujeto y objeto en el campo de los estudios literarios, clave que demanda la puesta en acción de toda una serie de perspectivas, no sólo la hermenéutica o la axiológica (predominantes en su análisis), y que reclama una concepción actualizada del empirismo. Creo que, como cualquier teoría, la de la literatura debe afrontar aquello que Carl G. Hempel (1965) formuló como el dilema del teórico; esto es, tiene que aclarar que su propósito va más allá del mero alzado de conexiones deductivas entre oraciones observacionales. Dicho en otros términos, tiene que comprometerse más allá de lo observable, más allá de lo dado - debe salir del territorio de la obviedad, diría Jonathan Culler-, puesto que si no lo hace convierte en paradójico el proceso de teorización y en innecesaria la propuesta teórica. Contra la determinación kantiana del conocimiento en tanto experiencia de un sujeto trascendental hay que considerar la variabilidad empírica del sujeto cognoscente en sus dimensiones psicológica, cultural, sociológica e histórica. O lo que es lo mismo, la relatividad de la construcción a priori de la realidad y la evasión del estricto reducto de la experiencia por parte de lo empírico. Pero del razonamiento de Fokkema destacaría su apelación tangencial a la noción de problema. El profesor holandés pensaba entonces en la actividad científica como un tríptico en el que la parte central representa la investigación en acto y la derecha culmina en la publicación y el debate público de los resultados, aunque lo que interesa en este punto es llamar la atención sobre la parte izquierda (que, no se olvide, en nuestra secuencialidad visual significa el punto de arranque). Ahí se representaría la selección de los problemas que van a ser sometidos a análisis e investigación ${ }^{7}$. Es el lugar, pues, de la pertinencia heurística, concepto que tiene dimensiones diversas, alguna estrictamente mecánica (en cuanto continuación de un proceso científico abierto), aunque también otras sociales, ideológicas, económicas y por supuesto también de género, colonialidad, etc.

Es pues epistemológicamente ingenuo, no será preciso reiterarlo mucho más, pensar que los objetos de estudio se nos den como algo en sí. En realidad, los señalamos y los analizamos en función de una posición teórica que nos permite verlos, identificarlos, considerarlos y tratarlos como pertinentes. Es la perspectiva, la theoria pues, lo que en definitiva problematiza el objeto de estudio y

\footnotetext{
${ }^{7}$ Mario Bunge y antes Nicolai Hartmann, como Althusser a su modo, han destacado el hecho de que la actitud problematizadora es la más característica de la racionalidad humana. Bunge ha escrito que la ciencia y la filosofía racionalista no son otra cosa que un estudio crítico de problemas, de modo que las tareas que competen al investigador son «tomar conocimiento de problemas que otros pueden haber pasado por alto; insertarlos en un cuerpo de conocimiento e intentar resolverlos con el máximo rigor y, primariamente, para enriquecer nuestro conocimiento» (Bunge, 1976: 191). A esa luz, añade, el investigador es el problematizador par excellence, pues liga el progreso del conocimiento con la capacidad para plantear, aclarar y resolver problemas. A su vez, Hartmann ha defendido en varios lugares de su obra un pensar problemático y aporético frente al pensar sistemático.
} 
demanda una observación atenta y crítica del mismo.

Ahora bien, siendo ese razonamiento impecable conviene complementarlo con otro que no siempre se tiene presente. La historia de la ciencia se encarga de ilustrar con suficientes ejemplos la existencia de sistemas teóricos que aspiraron a fundarse en una especie de impermeabilidad. Bajo diferentes prismas nos lo han recordado epistemólogos como Hempel, Popper, Kuhn, Lakatos, Toulmin o Prigogine. Desde otra perspectiva también Iuri Lotman, quien acuñó el término explosión para referirse precisamente al momento en que tal impermeabilidad se rasga. O Louis Althusser (1967) y Mario Bunge (1985) cuando analizaron, a distinta luz por supuesto, la relación entre ciencia e ideología.

Conviene distinguir por tanto entre objetos de estudio y problemas. Los primeros no se ven si no se quieren ver; los segundos siempre se hacen ver y oír. Aquellos son inertes desde un punto de vista epistemológico; estos otros, no. Ante todo porque entablan una dialéctica con la teoría que los señala, con la teoría a la que no cesan de interrogar y que contribuyen a conformar. A esta luz no es arriesgado defender que los problemas sí pueden disparar el mecanismo que pone en movimiento la teoría. Pero hay algo más. Los problemas, justo por su iluminación recíproca con la teoría, no se muestran aislados entre sí. Como postula la sociología del saber de Karl Mannheim, están asociados en constelaciones cuyo horizonte es histórico. A modo de ilustración de lo dicho sugiero considerar lo que ha significado y significa todavía la irrupción de un problema como el de la intermedialidad, hoy por hoy motor muy dinámico de diferentes aproximaciones teóricas y comparadas al fenómeno artístico-literario en una multiplicidad creciente de planos.

En el marco de la teoría literaria no sería tarea complicada señalar sectores, momentos o corrientes que en el sentido de Hartmann han tendido bien a un pensar sistemático constructivo bien a un pensar problemático investigador. La retórica grecolatina muestra como disciplina una vigorosa inclinación hacia el primero de esos modelos. La hermenéutica, al menos a partir del giro dialéctico de Schleiermacher, ha seguido el otro camino. Y de un modo semejante se podrían contraponer, por ejemplo, la sociología literaria de ortodoxia marxista y los estudios semiótico-culturales desarrollados por la Escuela de Tartu, o el New Criticism y el nuevo historicismo. Pero más que este alzado algo simplista de polaridades lo que me interesaría destacar en este punto es la opción favorable a la proporcionalidad entre esas dos formas de pensamiento que sin desequilibrio excesivo favoreciera el vector problematizador de la racionalidad. Una opción que supone ceder espacio a las estrategias de problematización. A mi modo de ver, quizás sea esta una de las notas más características de la actitud teórico-crítica dominante a día de hoy, decididamente empírica, des-ontologizada y muy pendiente de la crisis de legitimidad del conocimiento sometido a metarrelato tal y como fue referida por Lyotard en La Condition postmoderne ${ }^{8}$.

\footnotetext{
${ }^{8}$ Recuérdense las tres alegaciones principales surgidas a partir del pensamiento lyotardiano en relación con una teoría del conocimiento: 1) epistemología y ontología son indistinguibles, por lo que no habría objetos de estudio fuera del marco de conocimiento discursivo asumido; 2) no existe la posibilidad de una verificación empírica de la teoría; 3 ) verdad y ficción son indiscernibles, por lo que no hay diferencias fundamentales entre la ficción literaria y la ficción a la que se acogen los discursos filosófico o científico. Tomo el esquema de Patricia Waugh (1997: 152).
} 
Propongo acudir de nuevo a la sociología para entender en ese marco el significado profundo de lo que Bourdieu (1994) analizó como espace des possibles y lo que Sousa Santos (2002) promueve como urgente reacción contra la razón indolente a fin de consolidar un cambio de paradigma, no solo epistemológico. La razón indolente se manifestaría a la vez, siguiendo siempre al sociólogo portugués, como razón impotente (nada puede hacerse), como razón arrogante (nuestra racionalidad es incondicionalmente libre), como razón metonímica (la nuestra es la única racionalidad y no es preciso esforzarse en descubrir otras) y como razón proléptica (el futuro se limita a ser una superación lineal e infinita del presente). Las dos formas finales son las que mayor interés suscitan, motivo por el que Santos habilita dos críticas correlativas: una crítica de la razón metonímica y una crítica de la razón proléptica, destinadas entre otros objetivos justamente a descartar la posibilidad de persistencia de una gran teoría y su sustitución paradigmática por un trabajo de traducción destinado a fomentar la inteligibilidad mutua entre experiencias posibles y experiencias disponibles.

\section{Isegoría frente a logos tecnocrático}

En 1987, al presentar un panorama de la filosofía de la ciencia de los veinte años anteriores para situar después la evolución de la teoría social contemporánea, Anthony Giddens y Jonathan Turner hablaban de un cambio espectacular, que en el campo de las ciencias naturales se caracterizaría por una serie de rasgos encabezados por estos tres: el rechazo de la posibilidad de llevar a cabo observaciones teóricamente neutrales, la asunción generalizada de que el establecimiento de sistemas de leyes conectadas de forma deductiva ha dejado de funcionar como aspiración máxima de la investigación científica y, en especial, la instauración de la ciencia como tarea interpretativa.

Según lo destacado al comienzo de estas páginas, algo parecido ha ocurrido en la zona que aquí nos incumbe de forma directa, y a ello responden con precisión epígrafes como el no-lugar de la teoría literaria, empleado en varias ocasiones por Jenaro Talens, o nociones como atopía, que Túa Blesa (1999) ha ligado a una teoría por venir (y en convergencia con Blanchot o Derrida, también a una diferente actitud ante la lectura crítica), diferenciada de la propia de la tradición idealista por su aspiración al derrocamiento final de cualquier autoridad/origen/justificación cuya presencia sea percibida y asumida como luz (luz cegadora, puntualiza Blesa). Con ello converge la pregunta que encabeza el breve ensayo de Enric Sullà al que nos hemos venido refiriendo. En efecto: ¿y ahora qué hacemos?, ¿qué hacemos cuando, además, somos más que conscientes de que uno de los cambios fundamentales de nuestro tiempo afecta a aquel objeto - hoy otro siendo el mismo- que porfiamos en seguir denominando literatura? (Sullà, 2013: 138).

Recuérdese que en la apreciación de Walter Mignolo (1989: 65-66) las teorías de la literatura consolidadas durante los tres primeros cuartos del siglo XX habrían sido específicas y no genéricas, por cuanto se estatuyeron, según los casos, como teorías de la obra literaria, de la producción o la recepción literaria, de la excepcionalidad literaria, etc. Esta condición de especificidad habría facilitado la determinación de sus mutuas relaciones de dependencia y en definitiva agilizó algo parecido a una 
reducción conducente al espacio epistemológico de la literaturología $a^{9}$. Acaso porque la delimitación del modelo-objeto de estudio de su particular opción en aquellos momentos -la teoría del texto literario- se señalaba en el proceso de semiotización y no en otros marcos posibles más o menos restrictivos, Mignolo había defendido en otro trabajo la complementariedad de las comprensiones teórica y hermenéutica en el campo de los estudios literarios. Y entendió trasladable esa clasificación al campo de los estudios literarios al menos hasta su giro radical hacia una epistemología fronteriza, presentada por él como un paradigma otro (Mignolo, 2003). Tal epistemología, receptiva a los estudios poscoloniales y sobre la subalternidad, se orienta ya a la diversidad como proyecto y a la denuncia de todo universal abstracto que prolongue el que Mignolo considera proyecto agotado de la modernidad, fundamentado desde sus dimensiones culturales, socio-económicas y cognoscitivas en la colonialidad y en la poscolonialidad (la cual, en uno de sus sentidos, equivale a la colonialidad global). Desde el punto de vista discursivo, pragmático y argumentativo adquiere relevancia $-\mathrm{y}$ Mignolo resulta representativo en este punto de numerosos trayectos intelectuales paralelos al suyo- la incidencia en una geopolítica del conocimiento y la apuesta por un pensar/hablar entre lenguas, que conjuntamente implican una modificación significativa de lo que con Richard Rorty se puede entender como peso ético de la esfera de la conversación frente a la del conocimiento.

Pero la complementariedad de las comprensiones teórica y hermenéutica procede ya de las dificultades experimentadas por Aristóteles en la Poética, en concreto por lo que Lubomír Doležel (1990) entiende como falta de atención a la fundamentación epistemológica de la disciplina y de lo que estudia como incoherencias a la hora de optar por fijar o bien un marco descriptivo o bien un marco axiológico (tradición teórica / tradición crítica), viejo debate que ni siquiera sería posible cerrar aceptando la conocida propuesta de Aguiar e Silva (1998-1999: 89) al respecto, según la cual la teoría literaria es una epistemē mientras que la hermenéutica, como la retórica y la gramática, es una technē.

Suele reconocerse que toda teoría genera recursividad metateórica. En nuestro campo esta se ha dirigido con preferencia a los espacios no atendidos por una Poética históricamente volcada a la inspección de conceptos-guía (mímesis, género, belleza, ficción, decoro...) pero poco proclive, al menos hasta la disipación del clasicismo, a la concreción epistemológica. Y menos aún a lo que con Pierre Bourdieu (2001) podríamos llamar reflexividad o autoanálisis, es decir, a la exploración de los principios que rigen las prácticas académicas y de investigación propias contempladas no desde un punto de vista narrativo o biográfico sino meramente analítico-sociológico.

También en este sentido específico de la reflexividad y del equilibrio entre mirada teórica y comprensión, me parece representativo del movimiento general en los últimos veinte años el giro de Mieke Bal (1999) hacia un análisis cultural que mantiene algunos lazos con la historia cultural y con los estudios culturales pero que posee marcas ciertamente diferenciales, no exclusivas -insisto- de este programa de investigación. Sobre todo porque postula una nueva relación, que se declara de raíz

\footnotetext{
${ }^{9}$ Las teorías de tal disciplina cognoscitiva lo serían no de la literatura (enfocadas al dominio de estudio) ni literarias (pertenecientes a la literatura y no a su estudio) sino literaturológicas (discursos científicos producidos sobre el dominio de conocimiento literatura).
} 
dialógica y no instrumentalizadora, entre el sujeto que analiza y el objeto analizado. A través de esta surge una preterición de la historia y de toda filología reconstructiva. La alternativa aparece como una «memoria cultural en el presente». Se trataría, básicamente, de entender el pasado como una parte del presente en el momento en que aquel se muestra al analista (al analista y a su circunstancia actual, al analista y a sus lecturas o bagaje intelectual).

La reflexividad viene dada justo por la voluntad de destacar en el discurso teórico-crítico la presentidad del análisis cultural. Esto lleva a concebir el análisis como expository writing, porque en él se muestran el objeto analizado y el analista cultural en simultaneidad durante el proceso de concretar un discurso, en el que por cierto juegan un papel enriquecedor y no limitativo los específicos misreadings del lector-investigador. Un efecto del programa, recogido de manera explícita por Bal (2002: 286-323) y destacado como metaconcepto de su propuesta, es el ya mencionado de intimidad crítica $^{10}$, que algo tiene que ver con las condiciones de «manifiesta connivencia» y de complicidad a las que se han referido en una línea de pensamiento no exactamente concordante Frances Fortier, Jacqueline Chénard y Céline Leclerc (1998) a propósito de los vínculos que el pacto crítico posmoderno favorece con el objeto de análisis una vez que desiste de localizar su verdad ${ }^{11}$. Es evidente que al introducir estas referencias estamos yéndonos del emplazamiento de la teoría al de la crítica, cuyo ejercicio, además de ser necesariamente correlativo del teórico en el sentido razonado por Wellek y Warren en su exitoso manual de 1949 y de incorporar aun el oportuno ajuste de un horizonte comparatista $^{12}$ exigido por Darío Villanueva (1991), me parece probable que supla durante algún tiempo más la que en otra altura histórica y cultural fue constitución hegemónica de la teoría en el marco general de los estudios literarios.

Para terminar quisiera aludir, siquiera brevemente, al tercero de los vectores que a partir de una apreciación de Fokkema presentaba hace un instante. Se trata de las implicaciones extradisciplinares de la actividad teórica, en particular de aspectos como el ético, el educativo y el sociopolítico. Reflexividad, intersubjetividad, conciencia individual y conciencia histórica guardan estrecha interdependencia, como es bien sabido. La apertura del conocimiento tiene en nuestro tiempo un correlato directo en la asimilación progresiva de la propia pluralidad de los seres humanos. En este

\footnotetext{
${ }^{10}$ Su utilización inicial se debe a Gayatri Spivak en A Critique of Postcolonial Reason (1999).

${ }^{11}$ En qué sentido pueda entenderse este protocolo como una aspiración a la conciliación entre espacio privado y espacio público, y entre sus respectivos léxicos y bases argumentativas -algo que Rorty (1989) entiende irresoluble--, no es asunto para dictaminar en este lugar. Sobre el específico contrato de comunicación asociado a la intervención del investigador véase en cualquier caso una exposición reciente, ilustrativa incluso por el autoanálisis autorial ofrecido, en Charaudeau (2013). Para este analista del discurso el contrato de comunicación es entendible como «la relation qui s'établit entre l'identité sociale de celui qui parle (ou écrit), celle de son public, le rôle qu'il doit jouer et le type de discours qu'il doit tenir, le tout en fonction de la situation de communication dans laquelle il se trouve» (Charaudeau, 2013: $\$ 16$ ).

${ }^{12}$ Poco a poco más pendiente cada vez de lo que represente en la actualidad una literatura mundial y abierto a un fructífero debate sobre metodologías específicas, heurística, propedéutica e incluso sobre su didáctica. Véase al respecto la importante contribución de David Damrosch, coordinador del volumen Teaching World Literature (2009). Por la misma razón, y por su engarce con lo que pasaré a desarrollar de inmediato, coincido en la necesidad de una reorientación del comparatismo en el sentido postulado por Antoni Martí Monterde (2011: 437-439) en el cierre de su documentada historia intelectual de la Literatura comparada, disciplina que «podria refundar-se així com la més complexa i àmplia reflexió sobre la relació entre identitat $\mathrm{i}$ alteritat, en tan que relació intellectual, com a crítica de les identitats en la cruïlla entre el camp literari, el camp intellectual, el camp polític, el camp acadèmic i el camp del poder» (439).
} 
marco merece ser recuperado el concepto etopolítico de isegoría, que a mi juicio tiene inmediata aplicación al campo que ahora enfocamos. La isegoría, o igualdad en el derecho a pensar y enunciar un discurso propio, va mucho más allá de un tibio ejercicio de tolerancia pasiva. Se constituye de hecho como acto promotor del interés dialógico por la razón del otro, un interés no estratégico sino esencialmente comunicativo, y al tiempo habilita una reconsideración de las relaciones entre el individuo y la sociedad en conjunto, dotando de un nuevo impulso los ideales de igualdad y libertad, el ethos democrático, si se quiere. Tenemos así, respectivamente, el marco para una ética de la alteridad y para una ética de la aliedad.

Se recordará que al hablar de la conceptualización de la critical intimacy por parte de Mieke Bal, la presentamos como condición y efecto del análisis cultural. En realidad, se trataría de un efecto múltiple, dada su incidencia en órdenes distintos, entre ellos el intelectual, el social y el pedagógico. La lectura crítica de los textos y de la autoridad del autor es el efecto inmediato, por supuesto. Pero es igualmente importante la reconducción de la intimidad crítica, como quiere la propia Bal (2002: 283 290), hacia un dialogismo decididamente ajeno al autoritarismo y a la jerarquización interpersonal por renuncia a la objetualización en el terreno comunicativo y a la algo rancia distinción intelectual y moral del teórico. Al margen de la discusión abierta por Terry Eagleton (1983) cuando expuso los motivos por los que consideraba que ninguna teoría literaria podía dejar de ser una teoría política, me parece fuera de discusión que nuestra disciplina tiene un notable potencial de activación isegórica y de clarificación de la crisis de la razón, y que, al menos en ese sentido, posee también una clara repercusión pública y una tarea que cumplir en esta coyuntura de asfixia neoliberal. En primer lugar por ser, no sólo pero sí en buena medida, un análisis de las variantes del discurso. En segundo lugar, por su renuncia a convertirse en un mero aparato del logos tecnocrático y por constituirse ella misma en uno de los foros privilegiados en los que se discuten los componentes de la crisis ${ }^{13}$. Y finalmente por compartir con la propia literatura, como señaló en su momento Pozuelo Yvancos (1999), la capacidad de constituirse en espacio de complejidad hermenéutica donde las diferencias culturales no sólo se reconocen y comprenden sino que además se intercambian y discuten.

\section{Referencias bibliográficas}

Aguiar E SiLVA, V. (1998-1999): «As relações entre a Teoria da Literatura e a Didáctica da Literatura: filtros, máscaras e torniquetes», Diacrítica, 13-14, pp. 85-92.

Ahmad, A. (1992): In Theory: Classes, Nations, Literatures. Londres, Verso.

Althusser, L. (1967): «Sur le travail théorique. Difficultés et ressources», La Pensée, 132, pp. 3-22. Angenot, M. - J. Bessière - D. D. FokKema - E. Kushner, dirs. (1989): Théorie littéraire. Problèmes et perspectives. París, Presses Universitaires de France.

Aullón de Haro, P. (2013): Escatología de la crítica. Madrid, Dykinson.

BAL, M. ed. (1999): The Practice of Cultural Analysis: Exposing Interdisciplinary Interpretation.

\footnotetext{
${ }^{13}$ Véase al respecto el reciente e interesante «Manifeste pour les sciences sociales» de Calhoun y Wieviorka (2013).
} 
Stanford, Stanford University Press.

- (2002): Travelling Concepts in the Humanities: A Rough Guide. Toronto, University of Toronto Press.

BLEIKER, R. (2012): Aesthetics and World Politics. Londres, Palgrave Macmillan.

Blesa, T. (1999): «Atopías», Prosopopeya, 1, pp. 9-26.

Bourdieu, P. (1994): Raisons pratiques. Sur la théorie de l'action. París, Éditions du Seuil.

- (1997). Meditaciones pascalianas. Th. KAUF trad. Barcelona, Anagrama, 1999.

- (2001): Science de la science et réflexivité. París, Raisons d'Agir.

- (2003): Intelectuales, política y poder. A. B. Gutiérrez ed. y trad. Buenos Aires, Eudeba.

Bunge, M. (1976): La investigación científica. Su estrategia y filosofía. $5^{\mathrm{a}} \mathrm{ed}$. Barcelona, Ariel.

- (1985): Seudociencia e ideología. Madrid, Alianza.

Butler, J. (1997): Excitable Speech: A Politics of the Performative. Nueva York, Routledge.

Butler, J. - J. Guillory - K. Thomas, eds. (2000): What's Left of Theory: New Work on the Politics of Literary Theory. Nueva York, Routledge.

Butler, J. - E. LAClAU - S. ŽIŽEK (2000): Contingencia, hegemonía, universalidad. Diálogos contemporáneos en la izquierda. Buenos Aires, Fondo de Cultura Económica, 2004.

Calhoun, C. (1995): Critical Social Theory: Culture, History, and the Challenge of Difference. Oxford, Blackwell.

Calhoun, C. - M. WieviorkA (2013): «Manifeste pour les sciences sociales», Socio, 1, pp. 3-38, en http://socio.hypotheses.org/147 (última consulta, 23/6/2014).

Callus, I., - S. Herbrechter eds. (2004): Post-Theory, Culture, Criticism. Ámsterdam, Rodopi.

Campbell, D., - M. Schoolman, eds. (2008): The New Pluralism: William Connolly and the Contemporary Global Condition. Durham, Duke U. P.

CASAS, A. (2013): «Sabemos ver a emerxencia cultural? Achegas desde a socioloxía das ausencias e a socioloxía das emerxencias de Boaventura de Sousa Santos», en M. F. RodríguEZ PRAdo, ed., A emerxencia cultural a debate: referentes teóricos e aplicacións. Santiago de Compostela: Universidade de Santiago de Compostela, en prensa.

Charaudeau, P. (2009): «El contrato de comunicación en una perspectiva lingüística: convenciones psicosociales y convenciones discursivas», sitio Patrick Charaudeau - Livres, articles, publications, en http://www.patrick-charaudeau.com/El-contrato-de-comunicacion-en-una.html (última consulta, 23/6/2014).

- (2013): «Le chercheur et l'engagement. Une affaire de contrat», Argumentation et Analyse du Discours [en línea], 11, en http://aad.revues.org/1532 (última consulta, 23/6/2014).

CULleR, J. (1997): Literary Theory: A Very Short Introduction. Oxford, Oxford University Press.

DAmrosch, D., ed. (2009): Teaching World Literature. Nueva York, The Modern Language Association of America.

Debray, R. (1979): Le Pouvoir intellectuel en France. París, Ramsay.

Deleuze, G. - F. Guattari (1991): Qu'est-ce que la philosophie? París, Minuit. 
De Toro, F. ed. (1999): Explorations on Post-Theory: Toward a Third Space. Fráncfurt y Madrid, Vervuert \& Iberoamericana.

DoležEl, L. (1990): Poetics. Tradition and Progress. Lincoln, Nebraska U. P.

DUARTE, J. F. (1989): O espelho diabólico: construção do objecto da teoria literária. Lisboa, Editorial Caminho.

EAGLETON, T. (1983): Literary Theory: An Introduction. Oxford, Blackwell.

- (2004). Después de la teoría. R. GARCía PÉREZ, trad. Barcelona, Debate, 2005.

- (2012). El acontecimiento de la literatura. R. GARCÍA PÉREZ, trad. Barcelona, Península, 2013.

ELIAS, N. (1956): «Problems of Involvement and Detachment», British Journal of Sociology, 7, pp. 226-252.

Even-Zohar, I. (2010): Papers in Culture Research. $2^{\mathrm{a}}$ ed. Tel Aviv, Tel Aviv University.

Fortier, F. - J. ChÉNARD - C. LEClerC (1998): «Le Pacte critique postmoderne», en F. ForTIER, dir., «La Critique littéraire», Études littéraires, 30/3, pp. 13-31.

Fraser, N. (1992): «Rethinking the Public Sphere: A Contribution to the Critique of Actually Existing

Democracy», en C. Calhoun, ed., Habermas and the Public Sphere. Cambridge (Mass.), The MIT Press, pp. 109-142.

García CANCLINI, N. (2006): Diferentes, desiguales y desconectados. Mapas de la interculturalidad. Barcelona, Gedisa.

GidDens, A. - J. TuRnER eds. (1987): Social Theory Today. Londres, Polity Press.

GONZÁlez-Millán, X. (2000): Resistencia cultural e diferencia histórica. A experiencia da subalternidade. Santiago de Compostela, Sotelo Blanco.

GOULART, R. M. (2001): Literatura e Teoria da Literatura em tempo de crise. Braga, Angelus Novus. Guillory, J. (1993): Cultural Capital. The Problem of Literary Canon Formation. Chicago, University of Chicago Press.

GuSMÃO, M. (2004): «Da teoria como resistência da literatura», en C. MENDES DE SousA - R.

PATRício, orgs., Largo mundo alumiado. Estudos em homenagem a Vítor Aguiar e Silva. Braga, Universidade do Minho, vol. II, pp. 633-647.

Hempel, C. G. (1965): Aspects of Scientific Explanation and Other Essays in the Philosophy of Science. Nueva York, The Free Press.

Lodge, D. (1990): After Bakhtin. Essays on Fiction and Criticism. Londres: Routledge.

Martí Monterde, A. (2011): Un somni europeu. Història intellectual de la Literatura Comparada.

De la Weltliteratur a la Literatura Comparada. València: Universitat de València.

Mignolo, W. D. (1989): «Teorías literarias o teorías de la literatura. ¿Qué son y para qué sirven?», en G. REYES, ed., Teorías literarias en la actualidad. Madrid, El Arquero, pp. 41-78.

(2003): Historias locales / diseños globales. Colonialidad, conocimientos subalternos y pensamiento fronterizo. Tres Cantos, Akal.

PICORNELl, M. - M. PONS, eds. (2009): Literatura i cultura: aproximacions comparatistes. Palma:

Lleonard Muntaner. 
PozUelo Yvancos, J. M. (1999): «El conflicto de las Humanidades y la Teoría literaria: una retórica de la crisis», Prosopopeya, 1, pp. 91-114.

Provenzano, F. (2010). «La consécration par la théorie», COnTEXTES [en línea], 7, en http://contextes.revues.org/4629 (última consulta, 23/6/2014).

RANCIÈRE, J. (2000): Le Partage du sensible. Esthétique et politique. París: La Fabrique.

RORTY, R. (1989): Contingency, Irony, and Solidarity. Nueva York, Cambridge U. P.

- (1998): Achieving Our Country. Cambridge (Mass.), Harvard U. P.

Santos, B. de S. (2002): A crítica da razão indolente: Contra o desperdício da experiência, $4^{\mathrm{a}}$ ed. São Paulo, Cortez Editora.

SAPIRO, G. (2007): «Pour une approche sociologique des relations entre littérature et idéologie», COnTEXTES [en línea], 2, en http://contextes.revues.org/165 (última consulta, 23/6/2014).

SCHMIDT, S. J. (1997): «A Systems-Oriented Approach to Literary Studies». Canadian Review of Comparative Literature, 24/1, pp. 119-136.

SEnNeTt, R. (2006): The Culture of the New Capitalism. New Haven: Yale U. P.

SKINNER, Q., ed. (1985): The Return of Grand Theory in the Human Sciences. Cambridge: Cambridge U. P.

SPIVAK, G. C. (1999): A Critique of Postcolonial Reason: Toward a History of the Vanishing Present. Cambridge (Mass.), Harvard University Press.

(2003): Death of a Discipline. Nueva York, Columbia U. P.

Sullá, E. (1997): «El fet literari», en J. ABELLÁN - P. BALlART - E. SUllà, eds., Introducció a la Teoria de la Literatura. Manresa, Angle Editorial, cap. 2, pp. 45-68.

- (2013): «I ara, què fem? Breu historia de la Teoria (de la Literatura)», Els Marges, 100, pp. $133-138$.

TODOROv, T. (2007): La Littérature en péril. París: Flammarion.

VillanueVA, D. (1991): El polen de ideas. Teoría, crítica, historia y literatura comparada. Barcelona, Promociones y Publicaciones Universitarias.

Waugh, P. (1997): «The New Prometheans: Literature, Criticism, and Science in the Modern and Postmodern Condition», European Journal of English Studies, 1/2, pp. 139-164. 